\title{
Three-dimensional imaging of intracochlear tissue by scanning laser optical tomography (SLOT)
}

N. Tinne, L. Nolte, G. C. Antonopoulos, J. Schulze, J. Andrade, et al.

N. Tinne, L. Nolte, G. C. Antonopoulos, J. Schulze, J. Andrade, A. Heisterkamp, H. Meyer, A. Warnecke, O. Majdani, T. Ripken, "Threedimensional imaging of intracochlear tissue by scanning laser optical tomography (SLOT)," Proc. SPIE 9689, Photonic Therapeutics and Diagnostics XII, 96892H (29 February 2016); doi: 10.1117/12.2211066

SPIE. Event: SPIE BiOS, 2016, San Francisco, California, United States 


\title{
Three-dimensional imaging of intracochlear tissue by scanning laser optical tomography (SLOT)
}

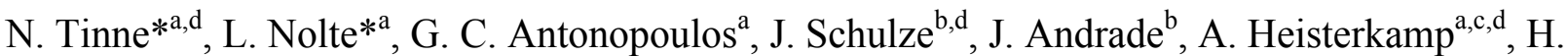 \\ Meyer $^{\mathrm{a}}$, A. Warnecke ${ }^{\mathrm{b}, \mathrm{d}}$, O. Majdani ${ }^{\mathrm{b}, \mathrm{d}}$ and T. Ripken ${ }^{\mathrm{a}, \mathrm{d}}$ \\ ${ }^{a}$ Laser Zentrum Hannover e.V., Biomedical Optics Department, Hannover, Germany; ${ }^{b}$ Hannover \\ Medical School, Department of Otorhinolaryngology, Hannover, Germany; ${ }^{\mathrm{c}}$ Gottfried Wilhelm \\ Leibniz Universitaet Hannover, Institute of Quantum Optics, Hannover, Germany; ${ }^{\mathrm{d}}$ Cluster of \\ Excellence "Hearing4all"
}

\begin{abstract}
The presented study focuses on the application of scanning laser optical tomography (SLOT) for non-destructive visualization of anatomical structures inside the human cochlea ex vivo. SLOT is a laser-based highly efficient microscopy technique, which allows for tomographic imaging of the internal structure of transparent large-scale specimens (up to $1 \mathrm{~cm}^{3}$ ). Thus, in the field of otology this technique is best convenient for an ex vivo study of the inner ear anatomy. For this purpose, the preparation before imaging comprises mechanically assisted decalcification, dehydration as well as optical clearing of the cochlea samples. Here, we demonstrate results of SLOT visualizing hard and soft tissue structures of the human cochlea with an optical resolution in the micrometer range using absorption and autofluorescence as contrast mechanisms.

Furthermore, we compare our results with the method of X-ray micro tomography (micro-CT, $\mu \mathrm{CT}$ ) as clinical gold standard which is based only on absorption. In general, SLOT can provide the advantage of covering all contrast mechanisms known from other light microscopy techniques, such as fluorescence or scattering. For this reason, a protocol for antibody staining has been developed, which additionally enables selective mapping of cellular structures within the cochlea. Thus, we present results of SLOT imaging rodent cochleae showing specific anatomical structures such as hair cells and neurofilament via fluorescence. In conclusion, the presented study has shown that SLOT is an ideally suited tool in the field of otology for in toto visualization of the inner ear microstructure.
\end{abstract}

Keywords: cochlea, inner ear, scanning laser optical tomography, tomography, SLOT, 3D microscopy, optical clearing, antibody staining, $\mu \mathrm{CT}$

\section{INTRODUCTION}

Scanning Laser Optical Tomography (SLOT) has been developed in 2011 as an advancement of optical projection tomography (OPT) [1]. It is a highly efficient 3D-microscopy technique, which is applicable for biological samples ranging from sub millimeter size up to several millimeters [2]. The system is able to use light extinction, scattering as well as fluorescence as contrast mechanisms. Hence, it is ideally suited for the fields of anatomy [2,3], neurobiology [4], and implant research [5] as has been proven.

In the field of otology, the anatomy and microanatomy of the human inner ear are of high interest. This knowledge is of great importance regarding the treatment of hearing disorders and hearing rehabilitation using cochlear implants as well as other auditory prosthesis. The cochlea consists of hard bony tissue on the one hand and soft tissue like thin membranes on the other. Here, the SLOT technique yields an advantage compared to the gold standard $\mu \mathrm{CT}$ [6]: It combines all contrast mechanisms of the light microscopy and, hence, enables visualization of biological structures with different optical properties. However, compared to confocal and multi-photon microscopy the advantage of SLOT is the visualization of transparent large-scale specimens (about $1 \mathrm{~cm}^{3}$ ) in toto.

Based on these prerequisites the aim of this study is to show that SLOT is an ideally suited instrument in the field of otology to tomographically visualize the complex inner ear microstructure.

*n.tinne@1zh.de / 1.nolte@1zh.de; phone +49 511 2788-229; fax +49 511 2788-100; www.lzh.de

Photonic Therapeutics and Diagnostics XII, edited by Bernard Choi, et al., Proc. of SPIE Vol. 9689, 96892H · @ 2016 SPIE · CCC code: 1605-7422/16/\$18 · doi: 10.1117/12.2211066 


\section{MATERIALS AND METHODS}

In this study, human cochlea samples have been analyzed to examine if SLOT is suited for visualization of the hard and soft tissue structures inside the inner ear. In particular, the same sample was imaged via $\mu \mathrm{CT}$ after decalcification to enable comparison with this gold standard method of otology. Furthermore, to verify the possibility of specific antibody staining in combination with SLOT imaging cochleae of a mouse were used. Hence, there were small differences in sample preparation between the two species. In Figure 1 a schematic overview of the procedures can be found; the single steps are described in detailbelow.

a)

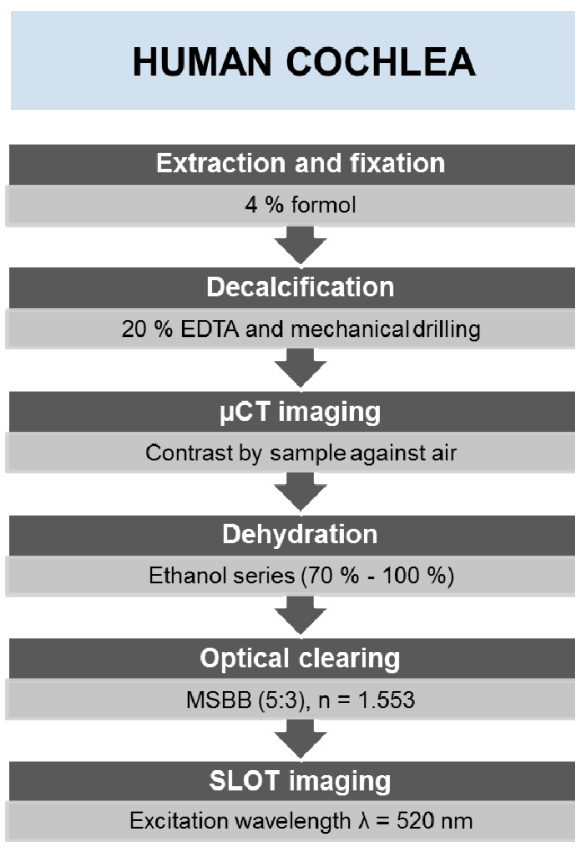

b)

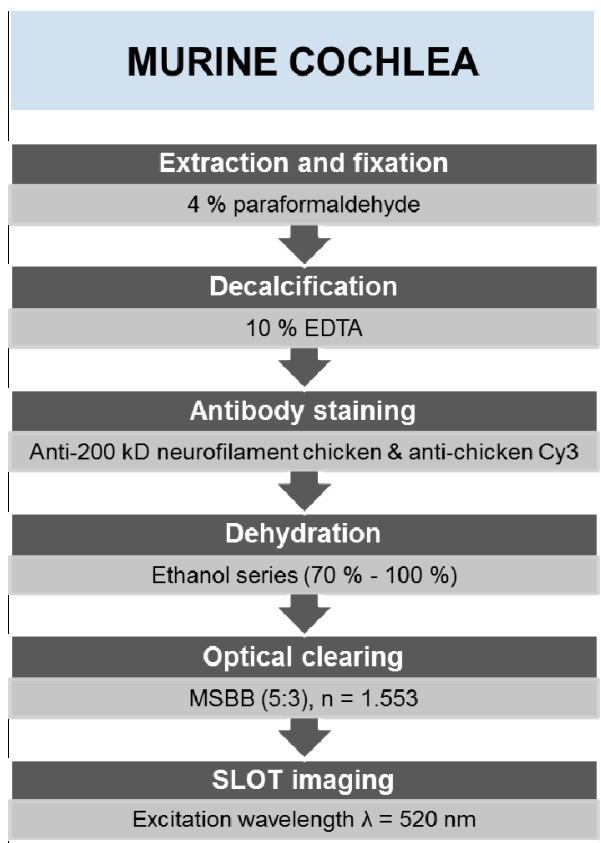

Figure 1. Schematic overview of the methods of sample preparation: a) human cochlea and b) murine cochlea.

\subsection{Chemical sample preparation}

Extracted cochleae were fixed using $4 \%$ formol for the human and $4 \%$ paraformaldehyde for the murine cochleae, respectively. While the following decalcification has been performed with $20 \%$ ethylenediaminetetraacetic acid (EDTA) in combination with mechanical drilling for the human samples, $10 \%$ EDTA was used for the much smaller murine cochleae.

In both cases, the samples were dehydrated via an ethanol series (increasing from $70 \%$ up to $100 \%$ ). Afterwards the ethanol was substituted by a mixture of five parts methyl salicylate and three parts benzyl benzoate (MSBB, 5:3). This solution has an index of refraction of about $\mathrm{n}=1.553$ and leads to an optical clearing of the cochleae.

\subsection{Antibody staining of the murine cochleae}

Cochlea samples of the mouse were incubated in image-iT-FX Signal Enhancer (Thermo Fisher Scientific) and blocking solution ( $1 \%$ Triton-X, $10 \%$ serum and $0.5 \%$ BSA in PBS) to reduce unwanted background fluorescence. As primary antibody anti-200 kD neurofilament chicken has been used to label neurons inside the sample in combination with antichicken Cy3 (Abcam/Jackson Immuno Research) as secondary antibody. 


\subsection{X-ray micro tomography}

The $\mu \mathrm{CT}$ imaging of the human cochlea has been performed with the "Phoenix Nanotom S" (General Electric Measurement \& Control, Frankfurt am Main, Germany). The measurement took about 4 hours and the tube parameters were $80 \mathrm{kV}$ and $60 \mu \mathrm{A}$.

\subsection{SLOT}

Scanning Laser Optical Tomography (SLOT) is Computer Tomography (CT) with visible laser light. The experimental setup is shown schematically in Figure 2. Here, a brief introduction to the principle and the experimental setup of SLOT will be given. A more detailed description can be found elsewhere [2,3].

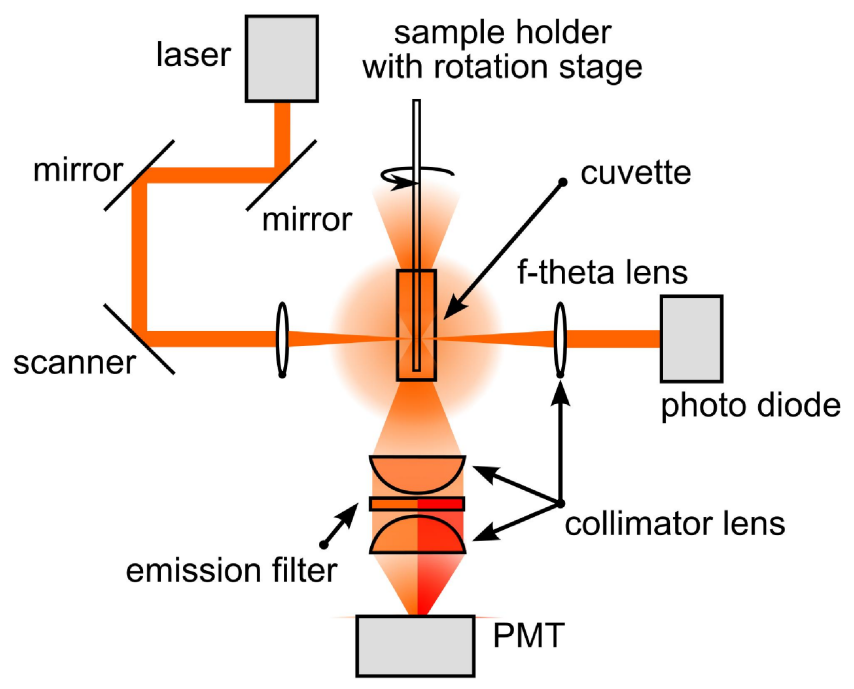

Figure 2. Schematic depiction of the experimental setup for Scanning Laser Optical Tomography (SLOT) (modified from [3]).

A laser diode is used as $\mathrm{cw}$ light source; available wavelength are $\lambda=405,445,520$ and $633 \mathrm{~nm}$ with a maximum power of $10 \mathrm{~mW}$. The light is coupled into the setup via single mode optical fiber. Afterwards, the light is collimated with a 10x motorized zoom lens to adjust the numerical aperture (NA) of the illuminating optical system. Using the combination of a two axis scanner and a telecentric f-theta lens the laser beam is scanned across the sample for acquisition of a projection image. The light is focused in the center of the sample, which is located inside a cuvette filled with a refractive index matching liquid (here MSBB 5:3). Additionally, the sample is clamped and connected to a rotation stage.

Behind the cuvette, transmitted light is collected with a lens and measured by a photodiode. Scattered light as well as fluorescence are collected perpendicularly by a fiber bundle and detected with a photomultiplier tube (PMT). Emission filters in front of the PMT allow selecting the spectrum entering the detector. Hence, the choice of optical filter decides whether scattering or fluorescence is detected. After the acquisition of each projection image, the sample is rotated by a small angle. For each angular position, another projection image is taken until a complete revolution of the sample is completed. Finally, the volumetric images are reconstructed digitally by applying the inverse Radon Transformation to the projection data. 


\section{RESULTS}

\subsection{Sample preparation and optical clearing}

Figure 3 and 4 show that the methods for decalcification of the cochlea samples worked well. There was no visibly difference before and after decalcification; the sample appears opaque (see Figure 3, left and middle). However, after matching the refractive index the murine and human cochlea sample are transparent as needed for SLOT imaging.

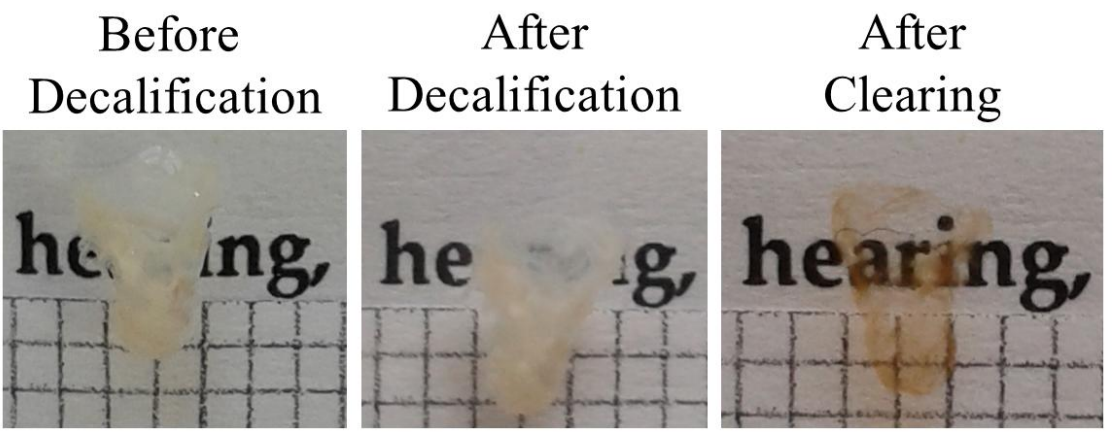

Figure 3. Photographs of a murine inner ear in liquid environment before decalcification (left), after decalcification (middle) and after optical clearing (right). Scale: see graph paper in the background.

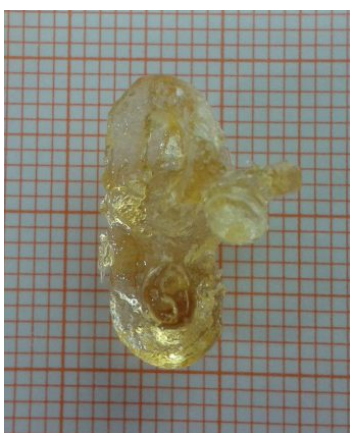

Figure 4. Photograph of a human inner ear after decalcification and optical clearing in air. Scale: see graph paper in the background.

\subsection{SLOT imaging of the human cochlea and comparison to $\mu \mathrm{CT}$ imaging}

The human cochlea sample has been imaged via $\mu \mathrm{CT}$ and SLOT. Two compare these two methods images which show a comparable plane of the sample are depicted in Figure 5. While the SLOT image is a superposition of two detection channels (extinction and autofluorescence) the $\mu \mathrm{CT}$ picture comprises only the absorption. It can be seen, that the SLOT image exposes more details of the hard as well as soft tissue structures. Furthermore, the system of ducts is not completely visible in the apical region using $\mu \mathrm{CT}$ which is perhaps due to liquid residuals inside the duct. 
a)

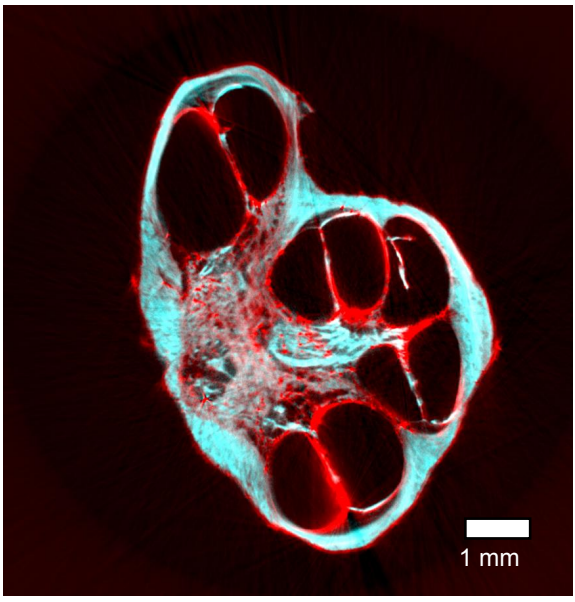

b)

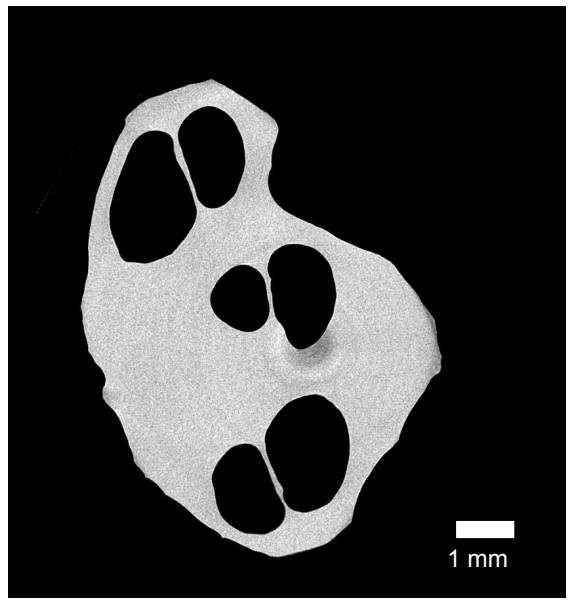

Figure 5. a) SLOT image of the human cochlea. Superposition of two detection channels: extinction (red) and autofluorescence (cyan). b) $\mu \mathrm{CT}$ image of the same sample in a comparable plane.

\subsection{Antibody staining and SLOT imaging of the murine cochlea}

The stained and cleared murine sample was imaged using SLOT. Figure 6 shows a maximum intensity projection of the reconstructed fluorescence channel. Due to the neurofilament antibody staining, the nerve fibers appear in a helical shape surrounding the modiolus. The surrounding signal is due to autofluorescence of the sample.

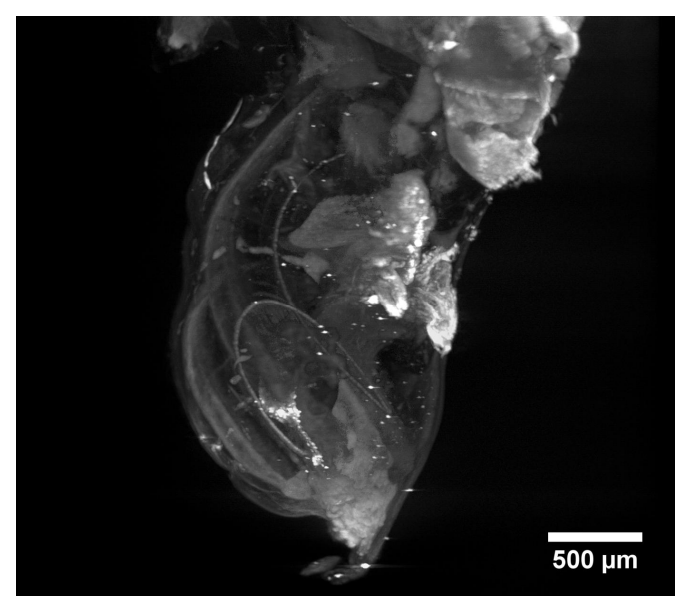

Figure 6. SLOT-fluorescence image of murine cochlea. The nerve fibers stained for $200 \mathrm{kD}$ appear as a helix surrounding the modiolus.

\section{CONCLUSION}

Scanning Laser Optical Tomography (SLOT) was shown to be an ideally suited 3D microscopy technique to visualize the inner ear microstructure in toto. The anatomy and microanatomy of the analyzed samples were clearly visible. Tissue structures could be differentiated via the multichannel superposition. Furthermore, a protocol for antibody staining has been developed and elicited using murine cochlea samples. This technique has the advantage to increase the signal of different anatomical structures of interest against the unwanted background signal. 


\section{ACKNOWLEDGEMENTS}

This work was supported by the DFG Cluster of Excellence EXC 1077/1 "Hearing4all" and the interdisciplinary research consortium "BIOFABRICATION for NIFE".

\section{REFERENCES}

[1] J. Sharpe, U. Ahlgren, P. Perry, B. Hill, A. Ross, J. Hecksher-Sørensen, R. Baldock, and D. Davidson, "Optical projection tomography as a tool for 3D microscopy and gene expression studies," Science 296, 541-545 (2002).

[2] R.-A. Lorbeer, M. Heidrich, C. Lorbeer, D. F. Ramírez Ojeda, G. Bicker, H. Meyer, and A. Heisterkamp, "Highly efficient 3D fluorescence microscopy with a scanning laser optical tomograph," Opt. Express 19, 5419-5430 (2011).

[3] R. Eickhoff, R.-A. Lorbeer, H. Scheiblich, A. Heisterkamp, H . Meyer, M. Stern, G. Bicker, "Scanning laser optical tomography resolves structural plasticity during regeneration in an insect brain", PLoS ONE 7(7): e41236. (2012)

[4] M. Kellner, M. Heidrich, R. Beigel, R. Lorbeer, L. Knudsen, T. Ripken, A. Heisterkamp, H. Meyer, M. Kühnel and Matthias Ochs, "Imaging of the mouse lung with scanning laser optical tomography (SLOT)," J Appl Physiol 113, 975-983 (2012).

[5] M. Heidrich, M. Kühnel, M. Kellner, R. Lorbeer, T. Lange, A. Winkel, M. Stiesch, H. Meyer and A. Heisterkamp, "3D imaging of biofilms on implants by detection of scattered light with a scanning laser optical tomograph," Biomed Opt Express 2, 2982-2994 (2011).

[6] A. Postnov, A. Zarowski, N. de Clerck, F. Vanpoucke, F. E. Offeciers, D. v. Dyck, and S. Peeters, "High resolution micro-CT scanning as an innovatory tool for evaluation of the surgical positioning of cochlear implant electrodes," Acta Oto-Laryngologica, 1-8 (2006). 